\title{
Muscle regeneration controlled by a designated DNA dioxygenase
}

\author{
Hongye Wang', Yile Huang ${ }^{2}$, Ming Yu ${ }^{3}$, Yang Yu', Sheng $\mathrm{Li}^{4}$, Huating Wang ${ }^{2,5}$, Hao Sun ${ }^{2,5}$, Bing Li $\mathbb{1}^{3}$, \\ Guoliang $\mathrm{Xu}^{6,7}$ and Ping $\mathrm{Hu}^{4,8,9}$
}

\begin{abstract}
Tet dioxygenases are responsible for the active DNA demethylation. The functions of Tet proteins in muscle regeneration have not been well characterized. Here we find that Tet2, but not Tet1 and Tet3, is specifically required for muscle regeneration in vivo. Loss of Tet2 leads to severe muscle regeneration defects. Further analysis indicates that Tet2 regulates myoblast differentiation and fusion. Tet2 activates transcription of the key differentiation modulator Myogenin (MyoG) by actively demethylating its enhancer region. Re-expressing of MyoG in Tet2 KO myoblasts rescues the differentiation and fusion defects. Further mechanistic analysis reveals that Tet2 enhances MyoD binding by demethylating the flanking $\mathrm{CPG}$ sites of $\mathrm{E}$ boxes to facilitate the recruitment of active histone modifications and increase chromatin accessibility and activate its transcription. These findings shed new lights on DNA methylation and pioneer transcription factor activity regulation.
\end{abstract}

\section{Introduction}

Skeletal muscles can regenerate due to the existence of muscle stem cells $(\mathrm{MuSCs})^{1,2}$. The normally quiescent MuSCs are activated after muscle injury and further differentiate to support muscle regeneration ${ }^{3,4}$. Skeletal muscle development and postnatal muscle regeneration are tightly regulated by muscle-specific transcriptional factors. $M y o D$ is considered to be the master regulator of myogenesis ${ }^{5}$, which recognizes and binds $\mathrm{E}$ box to activate transcription of target genes ${ }^{6-10}$. Another transcription factors Myogenin (MyoG) can also regulate myogenesis $^{11,12}$. MyoD directly activates the transcription of $M y o G$ by binding the E-box at its core promoter ${ }^{8}$. Despite the accumulating amount of excellent works about the mechanism of MyoD dependent transcription activation are still needed.

\footnotetext{
Correspondence: Ping Hu (hu_ping@grmh-gdl.cn)

${ }^{1}$ State Key Laboratory of Cell Biology, Shanghai Institute of Biochemistry and Cell Biology, Center for Excellence in Molecular Cell Science, Chinese Academy of Sciences, Shanghai 200031, China

${ }^{2}$ Department of Chemical Pathology, Li Ka Shing Institute of Health Sciences,

The Chinese University of Hong Kong, Hong Kong, China

Full list of author information is available at the end of the article

Edited by A. Stephanou
}

Ten-Eleven Translocation (Tet) family of DNA dioxygenases catalyze the active DNA demethylation and play critical roles in embryonic development, neural regeneration, oncogenesis, aging, and many other important biological processes ${ }^{13-19}$. There are 3 Tet DNA dioxygenase isoforms family in mammal, namely Tet1, Tet2, and Tet3. They share the highly conserved core catalytic domain at the $\mathrm{C}$ terminus. During early embryonic development, 3 Tets show functional redundancy ${ }^{19}$. In mammary tissues, Tet 2 is predominantly expressed over the other 2 isoforms and promotes luminal lineage commitment ${ }^{20}$. It has been considered that the specificity of Tet 2 functions is achieved by predominantly expressing one Tet isoform at a time. In the cases where all Tets are expressed simultaneously at the similar level, whether each Tet has non-redundant functions remains to be explored.

Demethylation of $M y o G$ promoter has been shown to contribute to the activation of MyoG transcription ${ }^{21-23}$. The functions of Tet2 in vivo during muscle regeneration and its mechanism remain to be further explored.

Here we found that Tet2, but not Tet1 and Tet3, specifically demethylated $M y o G$ enhancer. The Tet2 
mediated active DNA demethylation at the CpG sites near $E$ boxes enhanced the MyoD binding and increased the chromatin accessibility and active histone modification recruitment. These results revealed the specific functions of Tet 2 in vivo during muscle regeneration and shed new lights on understanding the mechanism of transcription regulated by DNA methylation.

\section{Results}

\section{Tet2 KO mice display muscle regeneration defects}

To identify the function of Tet 2 in muscle regeneration, we generated Tet 2 knockout mice by deleting exon 3 with homology recombination (Figs. 1A and S1A, B). Tet2 KO mice were born with normal skeletal muscle (Fig. S1C-G), suggesting that Tet2 is not required for muscle embryonic development and postnatal muscle growth.

We then induced muscle injury in Tet $2 \mathrm{KO}$ mice by CTX injection. The regeneration defects were observed as indicated by hematoxylin and eosin (H\&E) staining and decreased number of Myh3+ newly formed myofibers in Tet2 KO mice (Fig. 1B-D). The expression levels of $M y o G$ and $M y h 3$ were downregulated (Fig. $1 \mathrm{E}$ and F). The size of myofibers at the injury site was smaller than that in WT 28 days post injury (Fig. 1G-I) and the number of nuclei in each myofiber also decreased in Tet2 $\mathrm{KO}$ mice (Fig. 1J and $\mathrm{K})$.

We then generated Pax7CreERT2: Tet2 flox/flox mice, where Tet2 was knocked out specifically in MuSCs by tamoxifen induction (Fig. S2A). Phenocopying the constitutive Tet2 $\mathrm{KO}$, muscle regeneration defects and smaller myofiber size were observed (Fig. S2B-E). These results suggest that Tet $2 \mathrm{KO}$ mice display muscle regeneration defects, especially at the late stage of regeneration.

\section{Tet2 KO myoblasts display myotube fusion defects}

We isolated primary myoblasts from Tet $2 \mathrm{KO}$ mice for further examination. The number of MuSCs and the proliferation ability of Tet2 $\mathrm{KO}$ myoblasts were not affected both in vitro and in vivo (Fig. 2A, B and Fig. S3A-E). We then checked the differentiation ability of Tet2 KO myoblasts. Tet2 $\mathrm{KO}$ myoblasts formed thinner myotubes with lower fusion index (Fig. 2C-E). The expression level of muscle atrophy related genes $M A F b x$ (Atrogin 1) and Trim63 (Murf1) were unchanged, excluding the possibility of muscle atrophy (Fig. S4A and B) These results together suggest a potential fusion defects of Tet2 $\mathrm{KO}$ myoblasts.

To further confirm the fusion defects of Tet2 KO myoblasts, second phase fusion assays were performed as described $^{24,25}$. WT myoblasts were differentiated for 1 day and labeled with Cell Tracker Green. The mononucleated myoblasts isolated from either Tet2 KO or WT mice were labeled with Cell Tracker Red and co-cultured with the green WT myotubes (Figs. 2F and S4C). Both the size and the fusion index of myofibers decreased significantly after co-culturing with Tet2 $\mathrm{KO}$ myoblasts (Fig. 2G-J). Taken together, these results suggest that Tet2 $\mathrm{KO}$ myoblasts displayed fusion defects.

\section{Muscle differentiation related genes are downregulated in Tet2 KO myoblasts}

To further explore the mechanism, we performed mRNA sequencing using myoblasts isolated from Tet2 $\mathrm{KO}$ mice. Consistent with the differentiation defects in Tet2 KO mice, muscle cell differentiation, muscle contraction, and other muscle development related titles were enriched in the downregulated genes (Fig. 3A-C). Consistently, genes related to muscle cell differentiation and myotube fusion were all downregulated (Fig. 3D and G); the expression levels of fate determination and proliferation related genes remained to be unchanged (Fig. 3E); and there were some genes upregulated (Fig. 3F). These results combined suggest that Tet 2 regulates the expression of muscle differentiation and myotube fusion related genes.

\section{Muscle differentiation related genes are hypermethylated in Tet2 KO myoblasts}

Since Tet2 is an $\mathrm{mC}$ dioxygenase to remove DNA methylation, we next examined the DNA methylation status in Tet $2 \mathrm{KO}$ myoblasts by the whole-genome methylation sequencing. Every $2000 \mathrm{bp}$ in the genome was defined as a region. There were 8024 regions showing DNA methylation change (Fig. 4A). DNA hypermethylation regions were enriched in gene intron and intergenic regions (Fig. 4B), and related to many muscle differentiation associated genes (Fig. 4C).

We further looked for the overlapping genes with decreased expression level and hypermethylation regions in Tet2 KO myoblasts and found 38 genes (Fig. 4D and F). Genes related to muscle differentiation, myotube fusion, and maturation were enriched (Fig. 4F). There were 36 genes with both increased expression levels and hypomethylation (Fig. 4E and G). The bisulfite sequencing assays for the individual gene were performed to confirm the hypermethylation (Figs. $4 \mathrm{H}$ and $5 \mathrm{~B}$ ). These results suggest that Tet 2 could directly reduce the methylation level of the muscle differentiation related genes and therefore activate their expression.

\section{Tet2 activates MyoG expression by reducing the methylation level of E2 enhancer}

$M y o G$, a key myogenic regulator was among the above 38 genes. There was a 753 bp hypermethylation region located -13348 -12596 upstream of MyoG transcription starting site (TSS) (Fig. 5A). There were $13 \mathrm{CpG}$ sites within the $753 \mathrm{bp}$ DNA fragment (Fig. 5A). We divided the $753 \mathrm{bp}$ DNA fragment into 2 smaller fragments for 
A

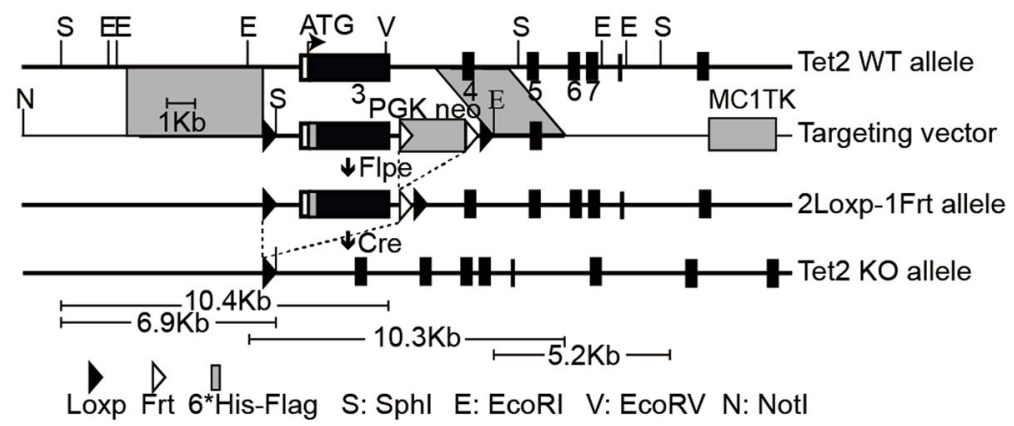

C

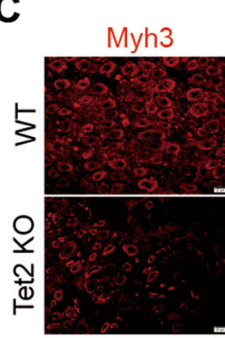

F

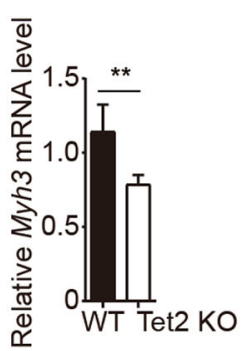

I

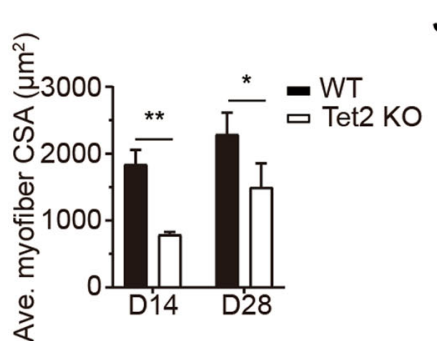

G

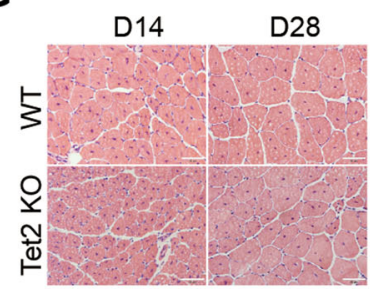

J

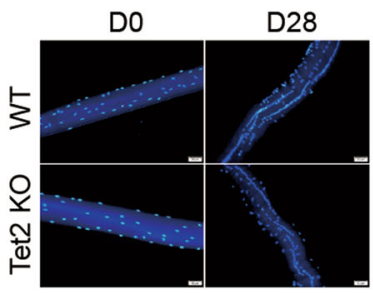

B

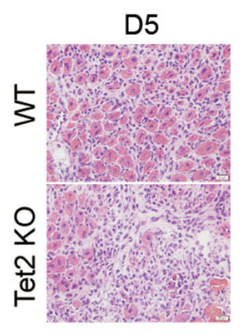

E

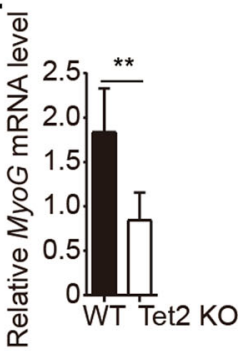

H

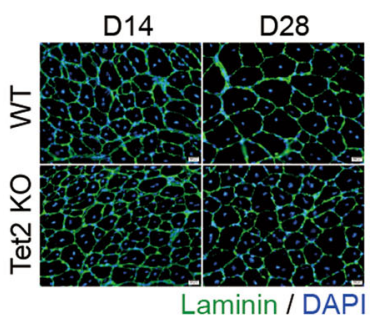

K

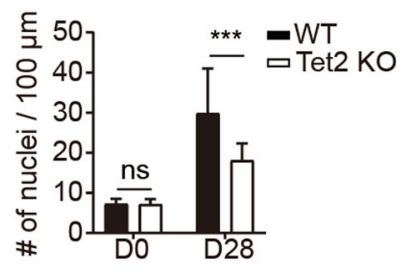

Fig. 1 Tet2 KO mice display muscle regeneration defects. A The scheme of Tet2 KO strategy. B Representative images of H\&E staining of TA muscles isolated from WT or Tet2 KO mice on day 5 after injury. Scale bars, $20 \mu \mathrm{m}$. C Immunofluorescence staining of Myh3 and Laminin of TA muscles isolated from WT or Tet2 $\mathrm{KO}$ mice on day 5 after injury. Scale bars, $20 \mu \mathrm{m}$. D Quantification of the number of Myh3+ myofiber per mm² on day 5 after injury $(n=3)$. E Relative mRNA expression level of MyoG in TA muscles isolated from WT or Tet2 KO mice on day 3 after injury $(n=5)$. F Relative mRNA expression level of Myh3 on day 5 after injury $(n=5)$. G Representative images of H\&E staining of TA muscles isolated from WT or Tet2 $\mathrm{KO}$ mice on day 14 or day 28 after injury. Scale bars, $50 \mu \mathrm{m}$. $\mathbf{H}$ Immunofluorescence staining of Laminin of TA muscles isolated from WT or Tet2 $\mathrm{KO}$ mice on day 14 or day 28 after injury. Scale bars, $20 \mu \mathrm{m}$. I Quantification of the cross-section area (CSA) from regenerated myofibers on day 14 and 28 after injury $(n=3)$. J Representative images of myonuclear staining of myofibers isolated from WT or Tet2 KO TA muscle before injury or on day 28 after injury. Scale bars, $50 \mu \mathrm{m}$. K Quantification of myonuclei number per $100 \mu \mathrm{m}$ in single myofiber on day 28 after injury $(n=3) .{ }^{*} p<0.05$, ${ }^{* *} p<0.01$, *** $p<0.001$.

further analysis. One $338 \mathrm{bp}$ fragment spanned -12596 $-12933 \mathrm{bp}$ region was named E1; the other $431 \mathrm{bp}$ fragment spanned $-12918 \sim-13348$ bp region was named E2 (Fig. 5A). Both E1 and E2 were hypermethylated in Tet2
KO myoblasts (Fig. 5B). Chromatin immunoprecipitation (ChIP) assays were performed to survey the histone marks marking the active enhancer. H3K4me1 and H3K27ac were enriched at both E1 and E2 (Fig. 5C), suggesting 
A

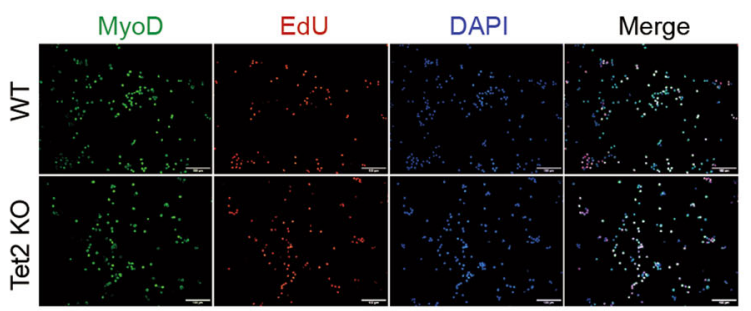

B

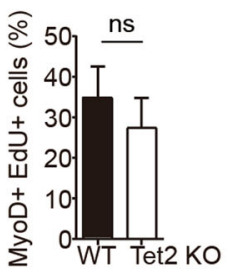

C

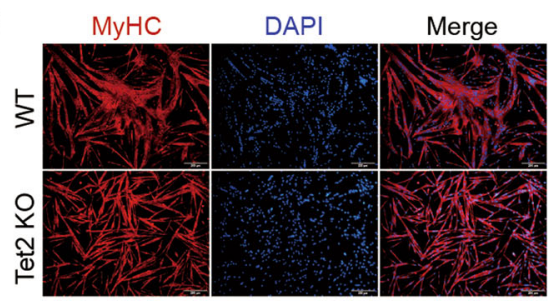

D

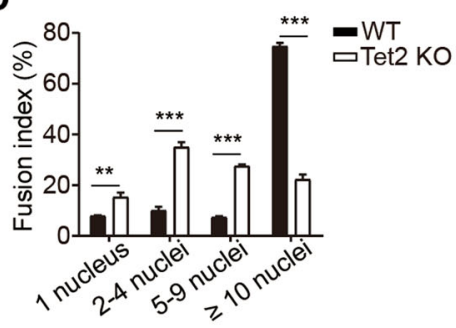

E

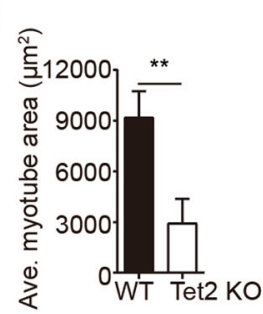

$\mathbf{F}$

Nascent myotubes Mononucleated (Differentiate for 1 day) myoblasts
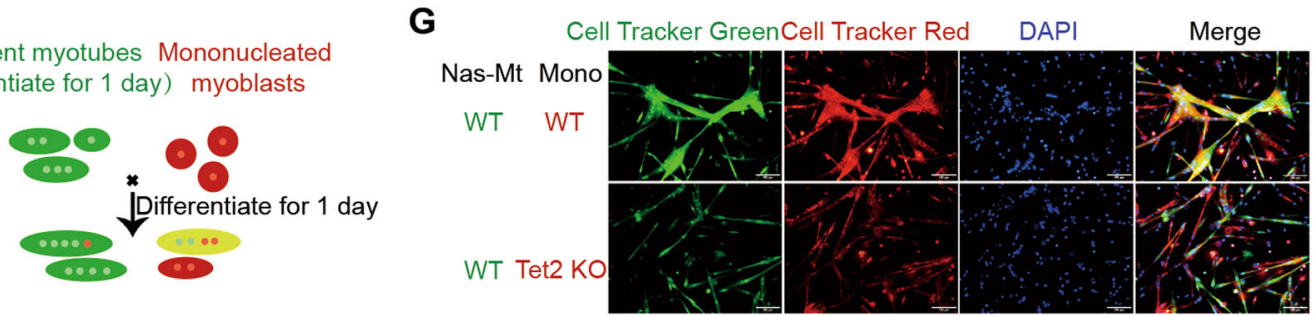

H

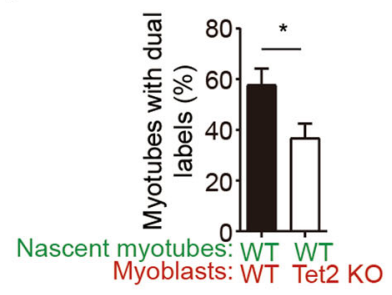

I

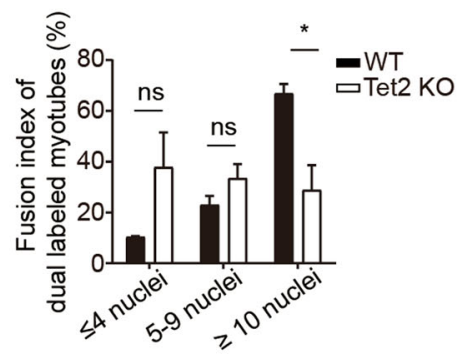

J

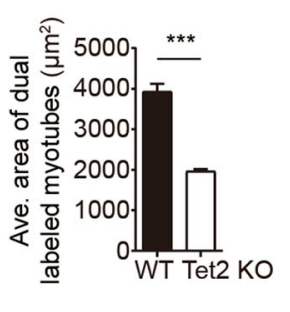

Fig. 2 Tet2 KO myoblasts display fusion defects in vitro. A Immunofluorescence staining of MyoD and EdU in myoblasts isolated from WT or Tet2 $\mathrm{KO}$ mice. Scale bars, $100 \mu \mathrm{m}$. B Quantification of the percentage of MyoD+ EdU+ myoblasts $(n=3)$. C Immunofluorescence staining of MyHC in differentiated myotubes. Scale bars, $200 \mu \mathrm{m}$. D Quantification of the fusion index in differentiated myotubes $(n=3)$. E Quantification of the average area in differentiated myotubes $(n=3)$. F Scheme of the second phase fusion assay. $\mathbf{G}$ Images of the second phase fusion assay results. Scale bars, $100 \mu \mathrm{m}$. H Quantification of the percentage of the dual labeled myotubes $(n=3)$. I Quantification of the fusion index of the dual labeled myotubes $(n=3)$. J Quantification of the average areas of dual labeled myotubes $(n=3) .{ }^{*} p<0.05,{ }^{* *} p<0.01,{ }^{* *} p<0.001$.

them to be enhancers for MyoG. E1 or E2 was inserted directly upstream of the $M y o G$ promoter to drive the expression of luciferase (Fig. 5D). The constructs were transfected to the primary myoblasts isolated from WT or Tet2 KO mice. In the WT myoblasts, E2 enhancer activated $M y o G$ transcription, while E1 enhancer barely worked (Fig. 5E). When the same construct was transfected to the primary Tet $2 \mathrm{KO}$ myoblasts, the activation of E2 enhancer was diminished (Fig. 5E). The Cs in E2 enhancer were randomly mutated to non-C nucleotides to abolish its ability to be methylated (Fig. 5F). The non- methylation mutant E2 enhancer showed higher transcription activation ability (Fig. 5G). These results together suggest that methylation of E2 enhancer by Tet2 down-regulates $M y o G$ transcription activity.

To confirm that MyoG is the major target of Tet2, $M y o G$ expression was knocked down in WT primary myoblasts (Fig. S5A and B). Similar to Tet2 KO myoblasts, the fusion index of $M y o G$ knocked-down cells decreased after differentiation (Fig. $5 \mathrm{H}$ and I). The expression of the similar set of genes was downregulated both in Tet2 $\mathrm{KO}$ cells and in MyoG knocked-down cells (Fig. 5J). When 
A

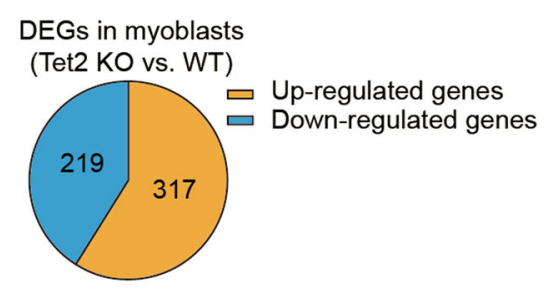

C

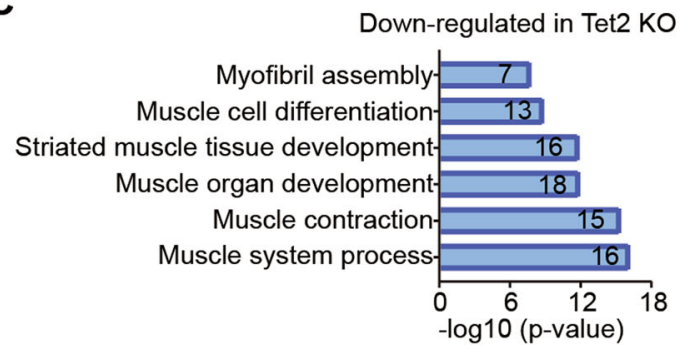

B

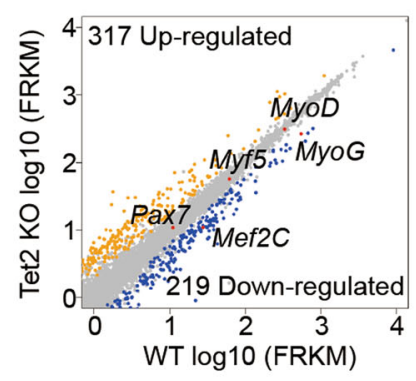

Up-regulated in Tet2 KO

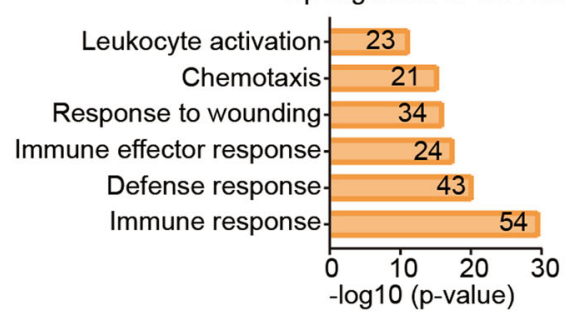

D

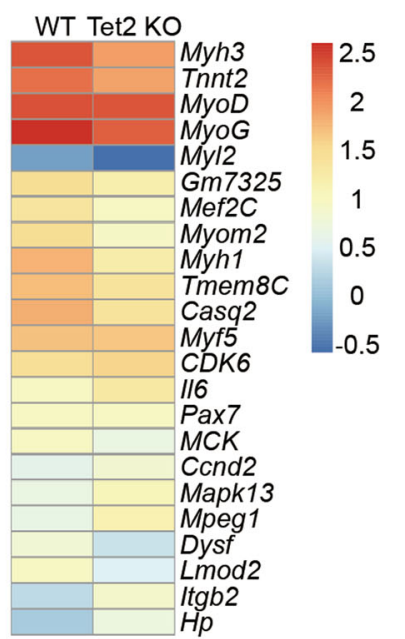

E

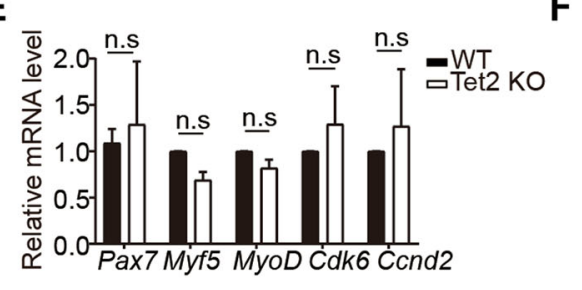

G

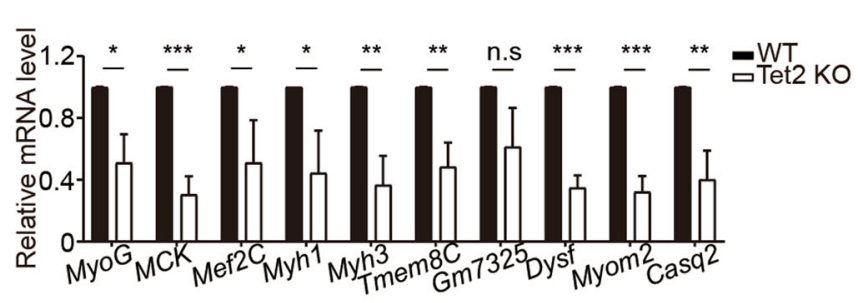

Fig. 3 RNA-sequencing analysis of Tet2 KO myoblasts. A Pie chart indicated the differentially expressed genes (DEGs) of Tet2 KO myoblasts compared to those of WT myoblasts. B Scatter plot indicated the DEGs of Tet2 KO myoblasts compared to those of WT myoblasts. C Gene ontology analysis of DEGs in Tet2 KO myoblasts. Numbers within bars indicated the number of genes identified within the corresponding gene ontology categories. $\mathbf{D}$ Heat map of the expression level of representative genes in myogenesis. $\mathbf{E}$ Expression levels of the fate determination and proliferation related genes in Tet2 $\mathrm{KO}$ myoblasts $(n=3)$. $\mathbf{F}$ Expression levels of the upregulated genes in Tet2 $\mathrm{KO}$ myoblasts $(n=3)$. G Expression levels of the differentiation related genes in Tet2 KO myoblasts $(n=3) .{ }^{*} p<0.05,{ }^{* *} p<0.01,{ }^{* * *} p<0.001$.

MyoG was re-expressed in Tet2 KO myoblasts (Fig. S5C), the fusion defect and the expression of the fusion related genes were rescued (Fig. 5K-M)), suggesting that $M y o G$ is the key target of Tet2 in muscle cells.

\section{Tet2 mediated DNA demethylation increases MyoD binding affinity at the neighboring $E$ boxes}

We next set out further mechanism exploration. ATAC-sequencing analysis was performed with myoblasts isolated from Tet2 $\mathrm{KO}$ or WT mice. The number of peaks decreased over 6 folds in Tet2 KO myoblasts, suggesting a significant decrease of chromatin accessibility with the absence of Tet2. The distribution of peaks was also changed in Tet2 $\mathrm{KO}$ myoblasts, especially at the enhancer region (Fig. 6A), suggesting the Tet2 dependent chromatin accessibility at the enhancer region. Consistently, the genes related to muscle cell differentiation also showed reduced chromatin accessibility in Tet2 KO myoblasts (Fig. S6A). The chromatin accessibility decreased dramatically around the E2 enhancer 
A

DMRs in myoblasts

(Tet2 KO vs. WT)

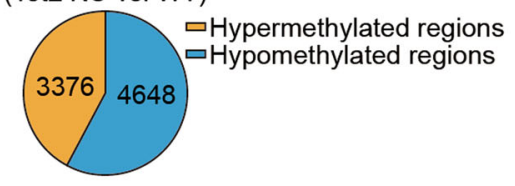

C

Hypermethylated in Tet2 KO myoblasts

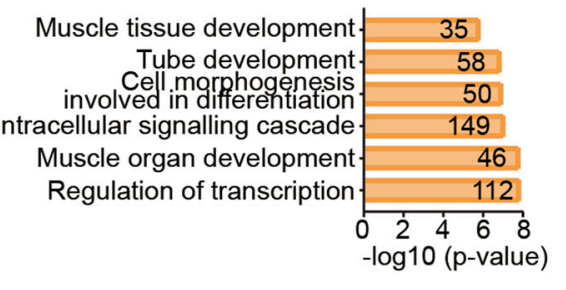

D

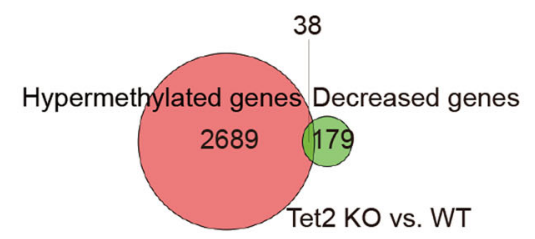

$\mathbf{F}$

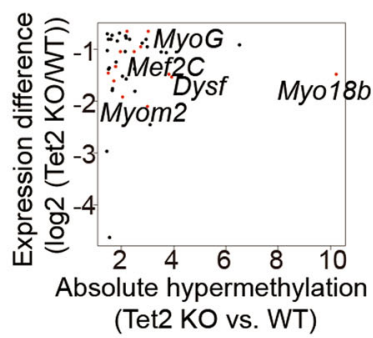

H

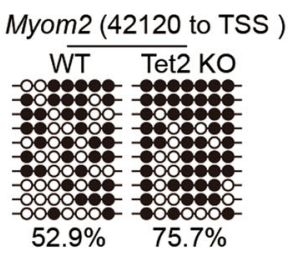

B

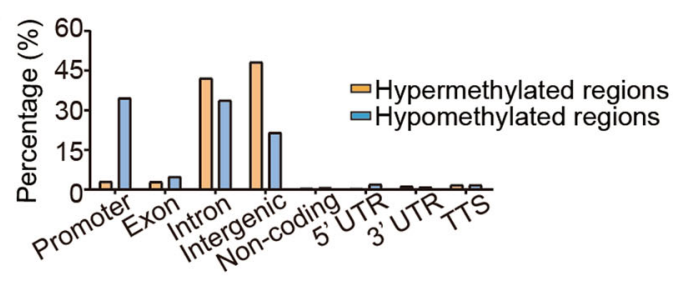

Hypomethylated in Tet2 KO myoblasts

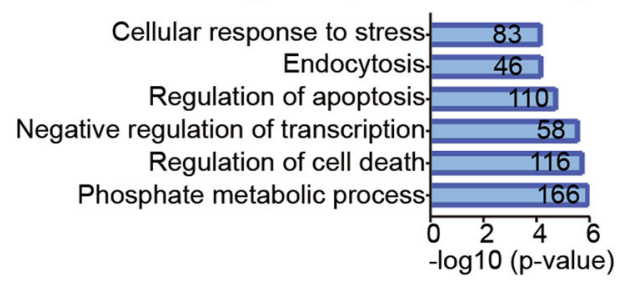

E

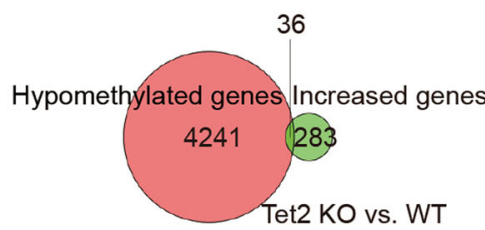

G

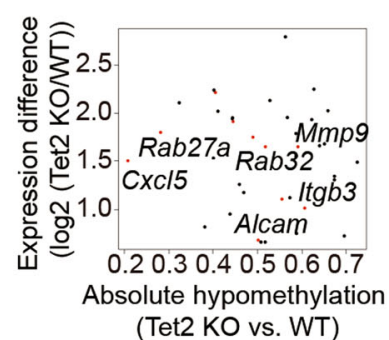

Fig. 4 The whole-genome methylation sequencing analysis of Tet2 KO myoblasts. A Pie chart indicated the differentially methylated regions (DMRs) of Tet2 KO myoblasts compared to those of WT myoblasts. B Distribution of the DMRs at various genomic features. C Gene ontology analysis of the DMRs. D Venn diagram of the hypermethylated and the downregulated genes in Tet2 KO myoblasts. $\mathbf{E}$ Venn diagram of the hypomethylated and the upregulated genes in Tet2 $\mathrm{KO}$ myoblasts. F Scatter plot of the genes hypermethylated and downregulated in Tet2 KO myoblasts. G Scatter plot of the genes hypomethylated and upregulated in Tet2 $\mathrm{KO}$ myoblasts. $\mathbf{H}$ The bisulfite sequencing results of Myom2 and Mef2C in WT or Tet2 KO myoblasts. White and black circles indicated hypomethylated and hypermethylated CpG sites, respectively.

hypermethylation region (Fig. 6B). In addition to the general decrease of chromatin accessibility in Tet2 KO myoblasts (Fig. S6B), some major peaks around the hypermethylated $\mathrm{CpG}$ sites were almost completely lost in E2 enhancer in Tet2 KO myoblasts (Fig. 6B, black arrows), suggesting that the chromatin accessibility at E2 enhancer is more sensitive to DNA hypermethylation. Furthermore, the levels of H3K4me1 and H3K27ac were downregulated at E2 enhancer in Tet2 $\mathrm{KO}$ myoblasts (Fig. 6C). Taken together, these results suggest that the increased DNA methylation at the enhancer region leads to reduced chromatin accessibility, decreased level of active histone marks, and declined transcription activity of MyoG.

$M y o D$ is the key transcription factor activating the transcription of $M y o G$ and inducing chromatin accessibility changes ${ }^{12,26}$. We then investigated the link between 


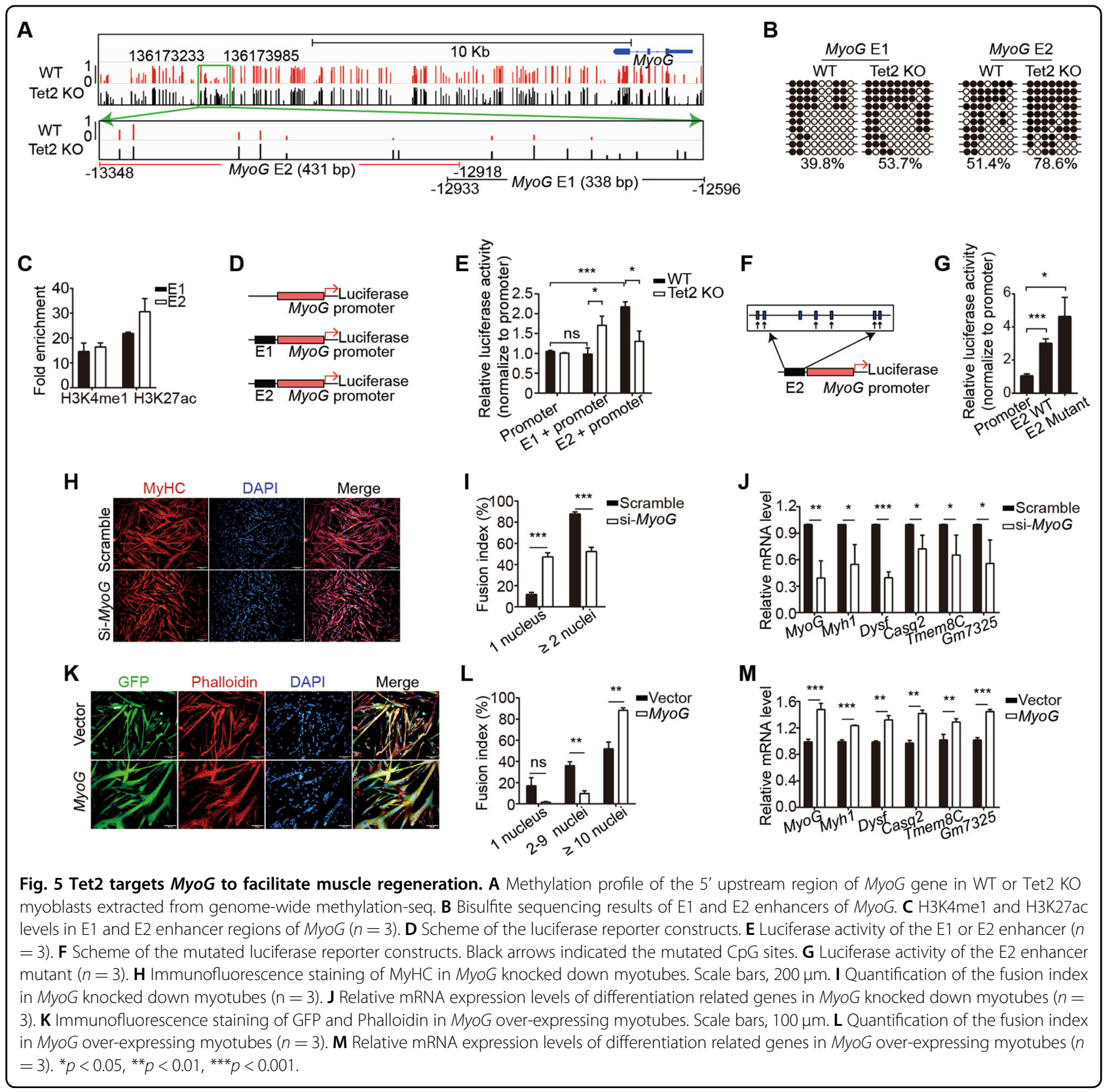

MyoD binding and Tet2. By sequencing analysis, we found 3 putative MyoD recognition sites at E2 enhancer (Fig. 6D). Interestingly, CpG sites were found within $10 \mathrm{bp}$ of the putative MyoD recognition sites (Fig. 6D). The recruitment of MyoD on E2 enhancer was surveyed by ChIP assays with a series of primers spanning the enhancer region (Fig. 6E). The binding of MyoD at E2 enhancer was reduced in Tet2 $\mathrm{KO}$ myoblasts (Fig. 6F), suggesting that the hypermethylation at the neighboring CpG sites represses MyoD binding.

We then performed bioinformatic analysis to identify the pattern of the methylation at the flanking CpG sites and MyoD binding in the whole-genome. By analyzing the
MyoD ChIP-seq data in myoblasts collected in both ENCODE and GEO (GEO number: GSM915186), we found that $\mathrm{CpG}$ sites, regardless of the methylation status, were located about $6-8$ bp upstream or downstream of $E$ boxes bound by MyoD (Fig. 6G). In sharp contrast, the methylated CpG sites were located 200-245 bp away from the $\mathrm{E}$ boxes bound by MyoD (Fig. $6 \mathrm{H}$ ), suggesting the loss of MyoD binding on sites with nearby methylated CpG sites.

We further surveyed the consensus sequence of $\mathrm{E}$ boxes in WT and Tet2 KO myoblasts. The top consensus sequence of MyoD in WT and Tet2 KO hypermethylation regions was the canonical CAGCTG sequence (Fig. S7). 


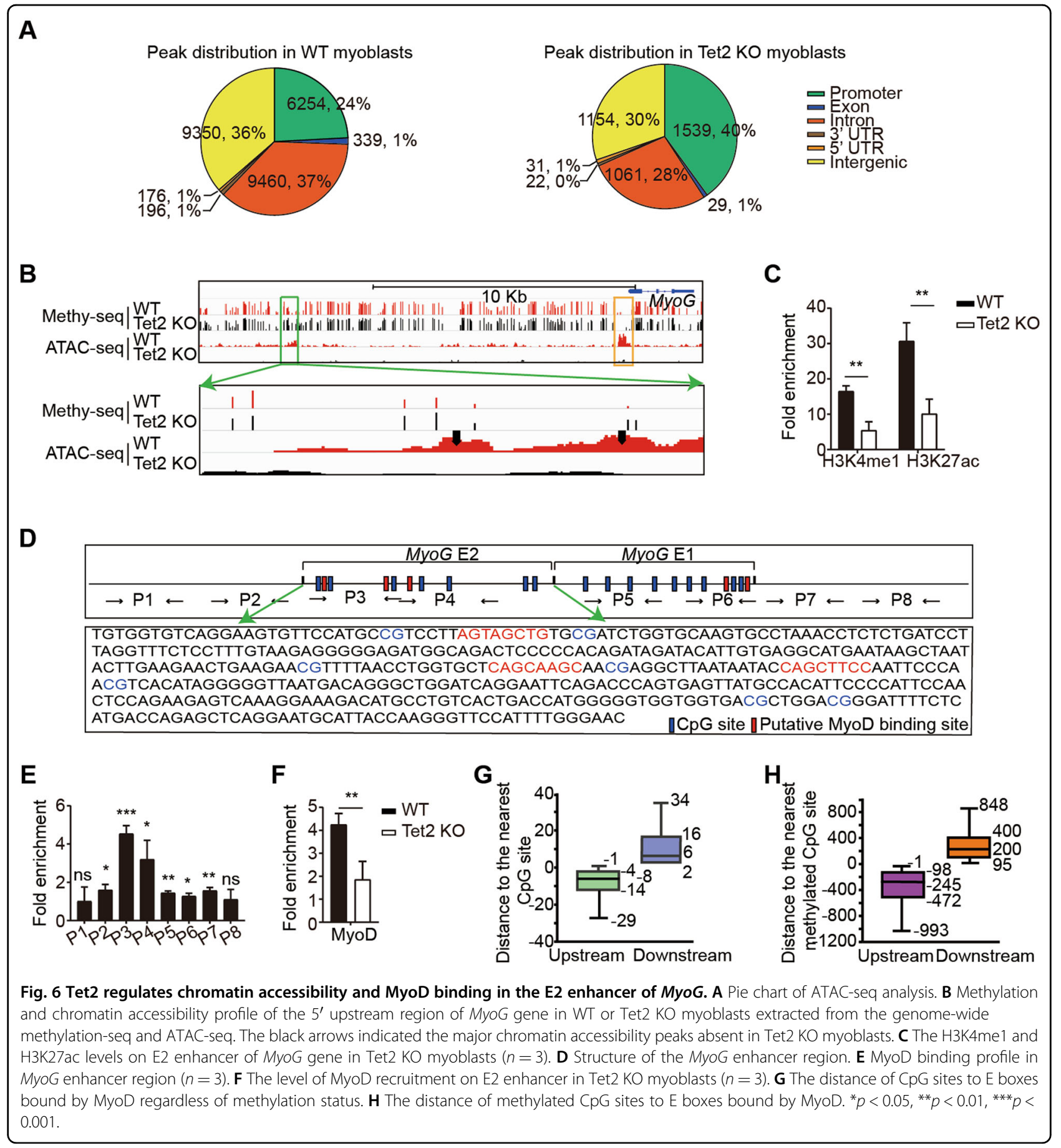

Some of the less prominent consensus sequences were absent in the hypermethylated regions, such as GGGAAR, CACACA, and AKAAAH (Fig. S7), suggesting that MyoD binding on these motifs are repressed by the neighboring CpG methylation. In contrast, some motifs like TTTAWW, CTGTGK, and CAGRTG were only present in the hypermethylated region, suggesting that the methylation of the flanking $\mathrm{CpG}$ sites improve MyoD binding. These results together suggest that the binding affinity of MyoD on E boxes is regulated by the methylation level of the neighboring CPG sites in a sequence dependent manner.

\section{Tet2 specifically promotes myoblast differentiation}

All 3 isoforms of Tet dioxygenases were upregulated during muscle regeneration process (Fig. 7A). We then 


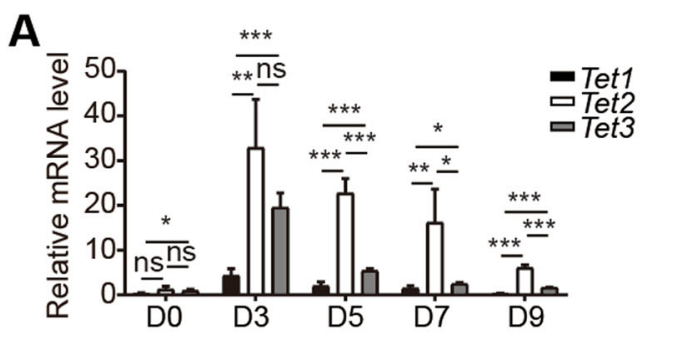

B
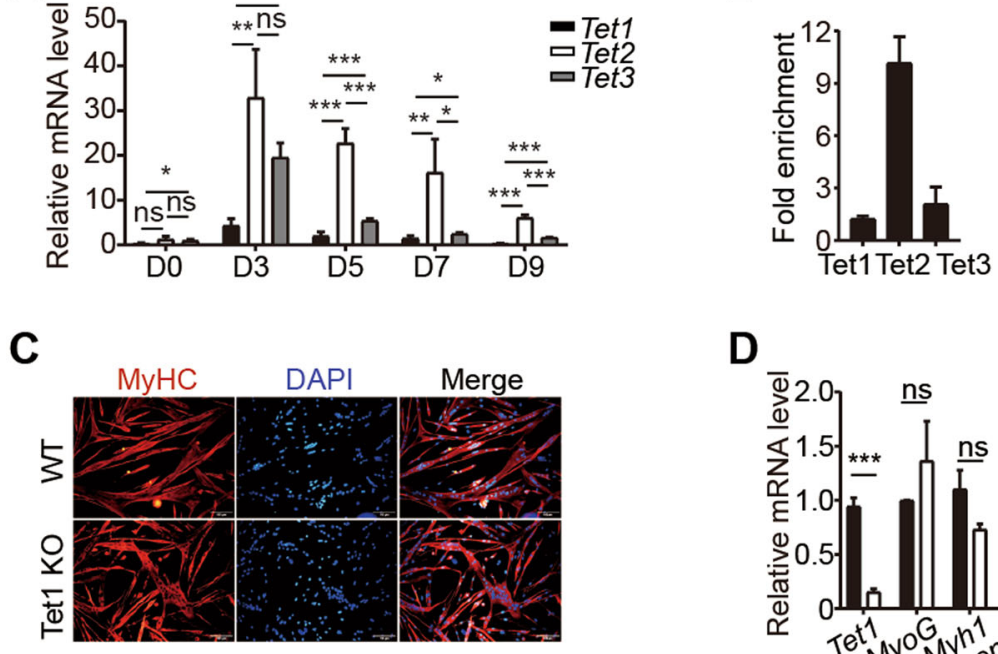

\section{E}

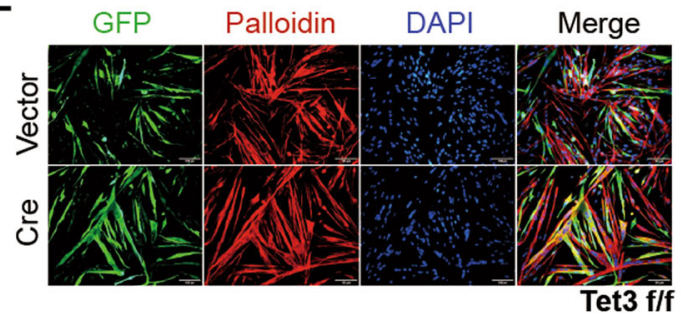

D

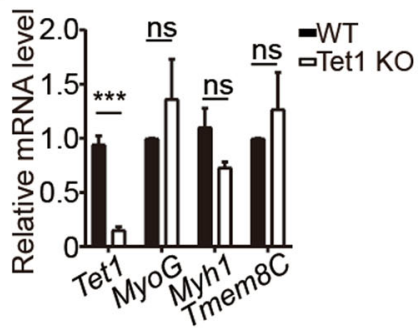

$\mathbf{F}$

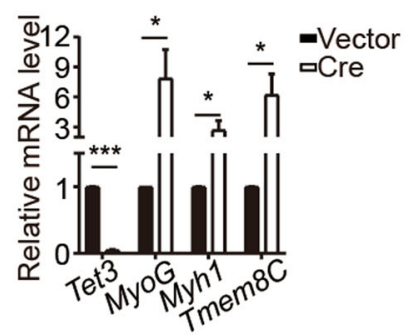

Fig. 7 Tet2, but not Tet1 and Tet3, specifically promotes myogenesis. A The relative expression levels of Tet1, Tet2, and Tet3 during muscle regeneration $(n=3)$. B Tet2 recruitment on E2 enhancer $(n=3)$. Myoblasts were isolated from Tet1-HA, Tet2-HA, Tet3-HA mice, respectively. ChIPqPCR assays were performed using anti-HA. C Immunofluorescence staining of MyHC in Tet1 KO myotubes. Scale bars, $100 \mu m$. D Relative mRNA expression level of Tet1, MyoG, Myh1, Tmem8C in Tet1 KO myoblasts $(n=3)$. E Immunofluorescence staining of GFP and Phalloidin in Tet3 KO myotubes. Scale bars, $100 \mu \mathrm{m}$. F Relative mRNA expression level of Tet3, MyoG, Myh1, Tmem8C in Tet3 KO myoblasts $(n=3) .{ }^{*} p<0.05,{ }^{* *} p<0.01,{ }^{* * *} p$ $<0.001$.

explored whether the promotion of myogenesis is a common function of all Tet dioxygenases. ChIP assays were performed in myoblast to survey the binding of Tet1, Tet2, and Tet 3 on E2. To avoid the variation caused by different antibodies, HA tag was knocked in at the C terminus of Tet1, Tet2, or Tet 3 mice, and the primary myoblasts were isolated. Surprisingly, only Tet2, but not Tet1 and Tet3, was enriched at the E2 enhancer (Fig. 7B), suggesting that Tet2 is specifically recruited to the E2 enhancer.

Differentiation ability of myoblasts isolated from Tet1 $\mathrm{KO}$ mice was checked. In contrast to Tet $2 \mathrm{KO}$, these cells differentiated normally and the expression of differentiation related genes remained unchanged (Fig. $7 \mathrm{C}$ and D). Tet3 KO mice are embryonic lethal ${ }^{27}$. We isolated myoblasts from Tet 3 flox/flox mice and infected them with adenovirus encoding Cre recombinases to knock out Tet3 (Fig. 7E). Unexpected, the Tet3 KO myoblasts showed enhanced differentiation ability and the differentiation related genes were upregulated (Fig. 7E and F). These results suggest that Tet2 regulates $M y o G$ transcription specifically and each Tet enzyme plays unique roles during skeletal muscle regeneration.

\section{Discussion}

The functions of the active demethylation in muscle regeneration in vivo have not been fully characterized. Here we report that Tet2 DNA dioxygenase, but not Tet1 and Tet3, specifically demethylates the CpG sites at the close approximation to $\mathrm{E}$ boxes to enhance MyoD binding and further facilitates the chromatin accessibility and recruitment of active histone modifications at the $M y o G$ enhancer to regulate muscle regeneration.

The transcription activity of MyoG has been implicated to show close ties with the DNA methylation level ${ }^{21,23}$. Tet2 has been shown to play an important role in regulating $\mathrm{C} 2 \mathrm{C} 12$ differentiation ${ }^{23,28,29}$. Here we further show that Tet2, but not Tet 1 and Tet 3 , specifically binds 
MyoG enhancer to activate $M y o G$ transcription, further emphasizing the importance of Tet2 in myogenesis. Compared to Tet1 and Tet3, Tet2 lacks the CXXC domain $^{17}$. CXXC domain has been suggested to direct Tet binding specificity ${ }^{30,31}$. The CXXC domain interaction achieved by protein-protein interactions between Tets and other proteins may attribute to the specific binding of each individual Tet isoform.

$M y o D$ is considered to be the "master transcription factor" and a pioneer factor to determine the muscle lineage ${ }^{6,7,32}$. ChIP-seq results reveal that there are thousands of constitutive MyoD binding irrelevant to transcription activation and muscle differentiation ${ }^{33}$. The specific activation of differentiation related genes by MyoD may require further regulation. Here we found that the demethylation of the flanking CpG sites within $100 \mathrm{bp}$ to $\mathrm{E}$ boxes increases the MyoD binding affinity. Our findings suggest that the methylation of the flanking region of the DNA elements is also the key element to regulate transcription factor binding affinity.

\section{Materials and methods \\ Animals}

Housing, mating and all experimental protocols for mice used in this study were performed in accordance with the guidelines established by the Institutional Animal Care and Use Committee in Shanghai Institute of Biochemistry and Cell Biology, Chinese Academy of Sciences. C57BL/6 were obtained from SLRC Laboratory Animal. Tet2 KO, Tet2 flox/flox, Tet1 KO, Tet3 flox/flox, Tet3HA mice were kindly provided by Dr. Guoliang Xu (Shanghai Institute of Biochemistry and Cell Biology, CAS). Tet1-HA and Tet2-HA tagged mice was kindly provided by Dr. Jingsong Li (Shanghai Institute of Biochemistry and Cell Biology, CAS). Pax7 CreERT2 mice were purchased from Jackson Laboratory (JAX, stock \#017763). Tet2 flox/flox mice were crossed with Pax7 CreERT2 mice to generate Pax7 CreERT2; Tet2 flox/flox mice. If not stated differently, 8-10-week-old male mice were used for all experiments.

Conditional knockout was induced by tamoxifen (Sigma) injection as described previously ${ }^{34}$. In brief, $10 \mathrm{mg} / \mathrm{ml}$ tamoxifen (Sigma) suspended in corn oil (Sigma) was injected intraperitoneally into 6-week-old Pax7-CreERT2: Tet2 flox/flox mice for 5 consecutive days at a dose of $100 \mathrm{mg} / \mathrm{kg}$ body weight per day. Littermates of the same genotype were injected with corn oil as a vehicle control.

\section{Antibodies}

Antibodies used for flow cytometry were AF700-antimouse Sca-1 (Thermo, 56-5981-82), PerCP/Cy5.5-antimouse CD11b (BD Biosciences, 550933), PerCP/Cy5.5anti-mouse CD31 (BD Biosciences, 562861), PerCP/
Cy5.5-anti-mouse CD45 (BD Biosciences, 550944), FITC anti-mouse CD34 (BD Biosciences, 553733), APC-antiIntegrin a7+ (R\&D, FAB3518A).

Antibodies used for western blots were Myogenin (F50D) (Santa Cruz, SC-12732), Flag-Tag (3B9) mAb (Abmart, M20008), GAPDH (14C10) Rabbit mAb (Cell Signaling, \#2118), Goat anti-mouse IgG-HRP (Santa Cruz, SC-2005), Goat anti-rabbit IgG-HRP (Santa Cruz, SC2004).

Antibodies used for immunofluorescence staining were anti-MyoD (Santa Cruz, sc-377460), anti-Pax7 (DHSB, RRID:AB_528428), anti-Myh3 (DHSB, F1.625), antiMyHC (Millipore, 05-716), anti-Laminin (Abcam, ab11575), anti-GFP (Aves Labs, GFP-1010).

Antibodies used for ChIP assays were anti-H3K4me1 (Abcam, ab8895), anti-H3K27ac (Abcam, ab4729), HA antibody (generated by our lab), mouse IgG (Abmart, B30010M), rabbit IgG (Abmart, B30011M).

\section{Cardiotoxin (CTX) injection}

Muscle injury was induced by intramuscular injections of CTX (Sigma-Aldrich, C3987) into TA muscle as previously described ${ }^{34,35}$. Each mouse was injected with $100 \mu \mathrm{l} 10 \mu \mathrm{M}$ CTX using 28-gauge needle at multiple injection sites in TA muscle. Mice were put under suction anesthesia with isoflurane during injection.

\section{$\mathrm{C} 2 \mathrm{C} 12$ cell culture}

$\mathrm{C} 2 \mathrm{C} 12$ cells were cultured at $37^{\circ} \mathrm{C}$ with $5 \% \mathrm{CO}_{2}$ in Dulbecco's modified eagle's medium (DMEM, Invitrogen) supplemented with $10 \%$ fetal bovine serum (FBS, Hyclone) and $100 \mathrm{U} / \mathrm{ml}$ penicillin/streptomycin (Invitrogen, 15140-122).

\section{Primary myoblasts isolation, expansion, and differentiation}

Primary myoblasts were isolated as previously described $^{35}$. Briefly, dissected TA muscles were digested with $10 \mathrm{ml}$ muscle digestion buffer (DMEM containing 1\% penicillin/streptomycin, $0.125 \mathrm{mg} / \mathrm{ml}$ Dispase II (Roche, 04942078001), and $10 \mathrm{mg} / \mathrm{ml}$ Collagenase D (Roche, 11088866001)) for $90 \mathrm{~min}$ at $37^{\circ} \mathrm{C}$. The digestion was stopped by adding $2 \mathrm{ml}$ of FBS. The digested cells were filtered through $70 \mu \mathrm{m}$ strainers. Red blood cells were lysed by $7 \mathrm{ml} \mathrm{RBC} \mathrm{lysis} \mathrm{buffer}\left(0.802 \% \mathrm{NH}_{4} \mathrm{Cl}, 0.084 \%\right.$ $\mathrm{NaHCO}_{3}, 0.037 \%$ EDTA in $\mathrm{ddH}_{2} \mathrm{O}, \mathrm{pH} 7.2-7.4$ ) for $30 \mathrm{~s}$, then filter through $40 \mu \mathrm{m}$ strainers. After staining with antibody cocktails (AF700-anti-mouse Sca-1, PerCP/ Cy5.5-anti-mouse CD11b, PerCP/Cy5.5-anti-mouse CD31, PerCP/Cy5.5-anti-mouse CD45, FITC antimouse CD34, APC-anti-mIntegrin a7+), the mononuclear cells were subjected for FACS analysis using Influx (BD Biosciences). The population of PI-CD45CD11b-CD31-Sca1- CD34+ Integrin a7+ cells was collected. 
Primary myoblasts were cultured on $0.5 \mathrm{mg} / \mathrm{ml}$ Type I collagen (Corning, 354249) coated dish as described previously $^{35}$ and differentiated in differentiation medium (DMEM containing $2 \%$ horse serum (Hyclone, SH30074.03) and $100 \mu / \mathrm{ml}$ penicillin/streptomycin (Hyclone, SV30010)) for $48 \mathrm{~h}$.

\section{Myofiber isolation}

Myofibers were isolated as described previously ${ }^{36}$. Briefly, TA muscles (uninjured and injured for 28 days) were isolated from tendon to tendon. The isolated TA was digested in DMEM medium (Gibco, 11965118) contain $0.2 \%$ collagenase D (Roche, 11088866001) for $90 \mathrm{~min}$ to disassociate myofibers. The digested muscle tissues were triturate three times with a wide bore $5 \mathrm{ml}$ pipet. Sit at room temperature for $5 \mathrm{~min}$ to allow fibers to settle down. Remove the supernatant and wash the myofibers with pre-warmed DMEM medium twice. Pick out single fibers with wide bore yellow tips and transfer directly to $4 \%$ paraformaldehyde to fix for $20 \mathrm{~min}$.

\section{Immunofluorescence staining}

TA muscle samples were embedded in OCT (Thermo Fisher Scientific, 6506), and frozen in liquid nitrogen for $20 \mathrm{~s}$ then cut for $8-10 \mu \mathrm{m}$ thick cryosections. Cultured cells or cryosections were fixed in $4 \%$ paraformaldehyde for $20 \mathrm{~min}$ at room temperature and washed 3 times with PBS. Samples were permeabilized with $0.5 \%$ Triton X-100 for $15 \mathrm{~min}$, and blocked in 3\% goat serum (Gibico, 16210072) for $60 \mathrm{~min}$ at room temperature. The samples were incubated in primary antibody for overnight followed by 3 washes with PBS. They were then incubated in fluorescent labeled secondary antibody followed by 3 washes with PBS and DAPI $(0.5 \mu \mathrm{g} / \mathrm{ml}$ ) (Invitrogen, D3571) staining. The mounted slides were visualized by BX53 microscope (Olympus).

Pax7 immunofluorescent staining was carried out as described previously ${ }^{34,35}$. Briefly, the cryosections were permeabilized with pre-chilled methanol for $6 \mathrm{~min}$ at $-20^{\circ} \mathrm{C}$ and blocked with M.O.M. Blocking Reagent (Vector, MKB-2213) for $2 \mathrm{~h}$. The primary and secondary antibody incubation steps were the same as described above.

\section{EdU labeling}

Primary myoblasts were labeled with $10 \mu \mathrm{M}$ EdU for $2 \mathrm{~h}$ at $37^{\circ} \mathrm{C}$ followed by fixation with $4 \%$ paraformaldehyde, permeabilized with $0.5 \%$ Triton $\mathrm{X}-100$, then reacted with Click-iT reaction cocktail (Invitrogen, C10338) and counterstained with Hoechst 33342 (Sigma, B2261). $50 \mu \mathrm{g} / \mathrm{g}$ body weight EdU (Sigma, 900584) was injected intraperitoneally for two consecutive days before sacrifice. EdU staining was performed with Click-iT EdU Cell Proliferation Assay kit (Invitrogen, C10338).

\section{Cell fusion experiment}

Cell fusion was measured as previously described ${ }^{24,25}$. MuSCs were differentiated for $24 \mathrm{~h}$ in differentiation medium (DMEM containing $2 \%$ horse serum and $100 \mu / \mathrm{ml}$ penicillin/streptomycin). The nascent myotubes were stained with $0.5 \mu \mathrm{M}$ Cell Tracker ${ }^{\mathrm{TM}}$ Green CMFDA Dye (Invitrogen, C2925) for $10 \mathrm{~min}$ at $37^{\circ} \mathrm{C}$. The mononucleated myoblasts were stained with $0.5 \mu \mathrm{M}$ Cell Tracker $^{\mathrm{TM}}$ Red CMTPX Dye (Invitrogen, C34552) for $10 \mathrm{~min}$ at $37^{\circ} \mathrm{C}$ and added to the primary myotubes to further differentiate for another $24 \mathrm{~h}$. The cells were fixed with $4 \%$ paraformaldehyde and visualized by BX53 microscope (Olympus).

\section{ChIP assay}

ChIP assays were performed as previously described $^{37,38}$. Briefly, crosslinking was performed in $1 \%$ formaldehyde for $10 \mathrm{~min}$ at room temperature, then stopped by $125 \mathrm{mM}$ glycine for $5 \mathrm{~min}$. Nuclei were isolated in cell lysis buffer $(50 \mathrm{mM}$ pH7.6 HEPEs, $10 \mathrm{mM} \mathrm{KCl}, 1.5 \mathrm{mM}$ $\mathrm{MgCl}_{2}, 1 \mathrm{mM}$ EDTA, $0.5 \mathrm{mM}$ EGTA, 0.5\% Triton X-100, add protease inhibitors ( $1 \mathrm{mM}$ PMSF, $1 \mathrm{mM}$ DTT) freshly) and chromatin was further extracted from the nuclei using nuclei lysis buffer $(50 \mathrm{mM}$ pH7.6 HEPEs, $1 \mathrm{mM}$ EDTA, $0.5 \mathrm{mM}$ EGTA, $1 \%$ Triton X-100, $0.1 \%$ deoxycholate, add protease inhibitors freshly). The chromatin was then sheared to $200-500 \mathrm{bp}$ by sonication (Bioruptor) and applied for antibody precipitation. Dynabeads ${ }^{\mathrm{TM}}$ Protein G (Invitrogen, 10004D) were used to capture the precipitated chromatin by antibody and followed by washes and reverse crosslinking. The purified DNA fragments were detected by qPCR. The fold enrichment were calculated against IgG ChIP-qPCR. Primers were listed in Table S1.

\section{RT-qPCR}

Total RNA was extracted by TRIzol Reagent (Thermo, 15596-018), and reverse transcription was performed using reverse-transcriptase M-MuLV (NEB, M0253L) to generate cDNA, followed by. qPCR analysis using SYRB Green qPCR Mix (Roche, A0001) by QuantStudio6 Flex (Thermo). The expression level of each gene was normalized to that of GAPDH. Primers were listed in Table S2.

\section{Measurement of myofiber size and fusion index}

The myofiber cross-section area was measured by Image Pro Plus software. At least 100 myofibers were measured for each sample. The number of regenerated myofibers and the fusion index (the number of nuclei in differentiated myotubes) were also counted by Image Pro Plus software. At least 3 fields were measured for each sample. The identity of the samples was blinded to the personnel who performed the measurement. 


\section{Statistical analysis}

The numbers of biological replicates and technical repeats in each experimental group were indicated in figure legends. For single myofiber staining, at least 10 single myofibers were isolated from each mouse. For myofiber CSA quantification, at least 100 myofibers from each mouse was quantified. For myoblasts proliferation and differentiation quantification, at least 3 microscope field were counted for each sample. Error bars represented standard deviation unless noted otherwise. Statistical differences between groups were determined by unpaired two-tailed $t$-test in GraphPad Prism 8 software. Statistical significance was set at $p<0.05$. ns indicated no significant difference, ${ }^{*}$ indicated $p<0.05,{ }^{* *}$ indicated $p<$ 0.01 , *** indicated $p<0.001$.

\section{RNA interference}

C2C12 myoblasts or primary myoblasts were transfected by siRNAs using Lipofectamine ${ }^{\mathrm{TM}}$ LTX reagent (Invitrogen, 15338030) following the manufacturer's instructions. At least 2 pieces of siRNA were used for each target gene.

siMyoG sense-1 $\left(5^{\prime}-3^{\prime}\right)$ : GCAUCACGGUGGAGGAUA UTT;

si MyoG antisense-1 $\left(5^{\prime}-3^{\prime}\right)$ : AUAUCCUCCACCGUGA UGCTT;

siMyoG sense-2 $\left(5^{\prime}-3^{\prime}\right)$ : GCAUGUAAGGUGUGUAAG ATT;

si MyoG antisense-1 $\left(5^{\prime}-3^{\prime}\right)$ : UCUUACACACCUUACA UGCTT;

siControl sense $\left(5^{\prime}-3^{\prime}\right)$ : UUCUCCGAACGUGUCACG UTT;

siControl antisense $\left(5^{\prime}-3^{\prime}\right)$ : ACGUGACACGUUCGG AGAATT.

\section{Luciferase reporter assay}

MyoG promoter (+3 -1596), MyoG promoter $+\mathrm{E} 1$ (WT or Mutation), and MyoG promoter + E2 (WT or Mutation) were cloned into pGL3 basic vector and transfected to primary myoblasts using the $\mathrm{Neon}^{\mathrm{TM}}$ Transfection System Starter Pack (Invitrogen, MPK5000S), respectively. After transfection 48 h, luciferase activities were measured using Dual-Luciferase Reporter Assay System (Promega, PR-E1910) by BioTek Synergy NEO (BioTek) following the manufacturer's instructions. Relative luciferase activity was calculated as the ratio of Firefly/Renilla luciferase activity. All experiments were repeated at least 3 times. Primers were listed in Tables S3 and S4.

\section{Conventional bisulfite sequencing}

Genomic DNA was extracted and treated with EZ DNA Methylation-Direct Kit (Zymo Research, D5005) according to the manufacturer's instructions. Bisulfite-treated
DNA was subjected to PCR amplification, then purified with the Gel Extraction Kit (TianGen, DP204) and cloned into pGEM-T vector system I (Promega, A3600). Individual clones were sequenced by standard Sanger sequencing. Data were analyzed by BISMA (http://services.ibc. uni-stuttgart.de/BDPC/BISMA/) ${ }^{39}$. Primers were listed in Table S5.

\section{RNA-seq}

Total RNA was extracted by TRIzol Reagent (Thermo, cat\# 15596-018) to constructed library using TruSeq RNA Library Preparation Kit v2 (Illumina, RS-122-2001). The raw RNA-seq reads were first evaluated by FastQC ${ }^{40}$ and preprocessed by Illumina universal adapter trimming and low-quality reads filtering with our in-house scripts ${ }^{41}$. All clean paired-end reads were then mapped to the mouse genome (mm9) using Tophat ${ }^{42}$ (version 2.0.13). Following alignment and filtering, gene expression levels were estimated by counting the number of fragments that are mapped to per kilobase of transcript per million mapped reads (FPKM). Differentially expressed genes (DEGs) between every pairs of $\mathrm{KO}$ and WT samples were analyzed with Cuffdiff ${ }^{43}$ (version 2.2.1) and genes with an adjusted $p$-value ( $q$-value) $<0.05$ were identified as DEGs.

\section{Whole-genome methylation-seq}

Genomic DNA was extracted and $1 \mu \mathrm{g}$ genomic DNA was treated with EZ DNA Methylation-Gold Kit (Zymo Research, D5005) according to the manufacturer's instructions. The methylation-seq library was constructed using TruSeq Nano DNA High Throughput Library Prep Kit (Illumina, 20015965). The bisulfite-treated DNA methylation sequencing data were analyzed by a data analysis pipeline called Methy-Pipe ${ }^{44}$. The paired-end reads were first aligned to the reference mouse genome (mm9) by the BSAligner module. The sequencing adaptors and low-quality bases on read ends were trimmed. All $C s$ in both the reference genome and sequenced reads were replaced by Ts in silico and the pre-processed and converted reads were aligned to the converted reference genome. Then methylation density (MD) calculation and the DMRs identification were performed by BSAnalyzer module. After MD calculation, a sliding window approach is employed and Mann-Whitney $U$ test is recruited to identify differentially methylated seed regions ( $p$-value $<0.01$ ). Regions with at least $20 \%$ changes of absolute methylation level and $p<0.01$ were defined as DMRs. Finally, we defined the merged seed regions with significant difference as putative DMRs.

\section{ATAC-seq}

ATAC-seq library was constructed from 5000 cells using TruePre DNA Library Pre Kit V2 for Illumina (Vazyme, TD501-01) and big peaks were filtered out using 
VAHTS DNA Clean Beads (Vazyme, N411-02) according to the manufacturer's instructions. These libraries were sequenced on Illumina HiSeq X Ten instrument with 150bp reads and paired-end parameter by WuXi NextCODE company. The $150 \mathrm{bp}$ paired-end ATAC-Seq reads were trimmed to $36 \mathrm{bp}$ paired-end reads and aligned to the mouse reference genome $(\mathrm{mm} 9)$ using Bowtie ${ }^{45}$ with parameters -X 2000 -no-mixed -no-discordant -local. Aligned reads are filtered for mapping quality $\geq 30$ and duplicate reads was removed by using the Picard MarkDuplicates (https://www.broadinstitutegithubio/picard/). Peak regions of each sample were called by software MACS2 $^{46}$ with options -f BAMPE -g mm -q 0.01. For visualization, the alignment bam files were converted into bedgraph files using homer (http://homer.ucsd.edu/ homer/ngs/ucsc.html). Differential peaks were adjusted by DESeq2 with fold change $>2$ and $p<0.05$.

\section{Acknowledgements}

We thank Drs. Fei Lan and Dangsheng Li for helpful discussions, the National Protein Science Center (Shanghai) for helps on FACS sorting, the cell biology facility of SIBCB for helps on imaging acquirement and FACS analysis.

\section{Author details}

'State Key Laboratory of Cell Biology, Shanghai Institute of Biochemistry and Cell Biology, Center for Excellence in Molecular Cell Science, Chinese Academy of Sciences, Shanghai 200031, China. ${ }^{2}$ Department of Chemical Pathology, Li Ka Shing Institute of Health Sciences, The Chinese University of Hong Kong, Hong Kong, China. ${ }^{3}$ Department of Biochemistry and Molecular Cell Biology, Shanghai Jiao Tong University School of Medicine, Shanghai 200233, China.

${ }^{4}$ Xinhua Hospital affiliated to Shanghai Jiao Tong University School of Medicine, Shanghai 20023, China. ${ }^{5}$ Department of Orthopaedics and Traumatology, Li Ka Shing Institute of Health Sciences, The Chinese University of Hong Kong, Hong Kong, China. ${ }^{6}$ State Key Laboratory of Molecular Biology, Shanghai Institute of Biochemistry and Cell Biology, Center for Excellence in Molecular Cell Science, Chinese Academy of Sciences, Shanghai 200031, China. ${ }^{7}$ Key Laboratory of Medical Epigenetics and Metabolism, Institutes of Biomedical Sciences, Medical College of Fudan University, Shanghai 200032, China. ${ }^{8}$ Max-Planck Center for Tissue Stem Cell Research and Regenerative Medicine, Bioland Laboratory (Guangzhou Regenerative Medicine and Health GuangdongLaboratory), Guangzhou 510005, China. ${ }^{9}$ Institute for Stem Cell and Regeneration, Chinese Academy of Sciences, Beijing 100101, China

\section{Author contributions}

P.H. and H.W. performed study concept and design; P.H. and H.W. performed development of methodology and writing, review and revision of the paper; $\mathrm{H}$. W. proved acquisition, analysis and interpretation of data, and statistical analysis; Y.H., H.W., and H.S. analyzed RNA-seq, the whole-genome methylation-seq, and ATAC-seq data. Y.Y., M.Y., and S.L. contributed Fig. S1A and Fig. S5B, C. G.X. and B.L. provided technical and material support. All authors read and approved the final paper.

\section{Funding}

This work was supported by the Strategic Priority Research Program of the Chinese Academy of Science (XDA16020400 to P.H.), Ministry of Science and Technology of China (2017YFA0102700 to P.H.), the National Natural Science Foundation of China (91649104 and 31671536 to PH; 31872817 and 32030019 to BL), Space Medical Experiment Project of China Manned Space Program (HYZHXM01017 to P.H.), National Key R\&D Program of China (2018YFC1004500 to $\mathrm{BL})$.

\section{Data availability}

All the sequencing data (mRNA-seq, methylation-seq, ATAC-seq) in WT and Tet2 KO primary myoblasts are available through GEO under the accession number GEO: GSE158649.

\section{Competing interests}

The authors declare no competing interests.

\section{Ethics statement}

Our study did not use human samples. All animal experiments were approved by the Animal Care and Use Committee in Shanghai Institute of Biochemistry and Cell Biology, Chinese Academy of Sciences.

\section{Publisher's note}

Springer Nature remains neutral with regard to jurisdictional claims in published maps and institutional affiliations.

Supplementary information The online version contains supplementary material available at https://doi.org/10.1038/s41419-021-03817-2.

Received: 24 February 2021 Revised: 27 April 2021 Accepted: 7 May 2021 Published online: 25 May 2021

References

1. Murphy, M. M., Lawson, J. A., Mathew, S. J., Hutcheson, D. A. \& Kardon, G. Satellite cells, connective tissue fibroblasts and their interactions are crucial for muscle regeneration. Development 138, 3625-37 (2011).

2. Feige, P., Brun, C. E., Ritso, M. \& Rudnicki, M. A. Orienting muscle stem cells for regeneration in homeostasis, aging, and disease. Cell Stem Cell 23, 653-64 (2018).

3. Brack, A. S. \& Rando, T. A. Tissue-specific stem cells: lessons from the skeletal muscle satellite cell. Cell Stem Cell 10, 504-14 (2012).

4. Fu, X., Wang, H. \& Hu, P. Stem cell activation in skeletal muscle regeneration. Cell Mol. Life Sci. 72, 1663-77 (2015).

5. Berkes, C. A. \& Tapscott, S. J. MyoD and the transcriptional control of myogenesis. Semin. Cell Dev. Biol. 16, 585-95 (2005).

6. Blackwell, T. K. \& Weintraub, H. Differences and similarities in DNA-binding preferences of $\mathrm{MyOD}$ and $\mathrm{E} 2 \mathrm{~A}$ protein complexes revealed by binding site selection. Science 250, 1104-10 (1990).

7. Shirakata, M. \& Paterson, B. M. The E12 inhibitory domain prevents homodimer formation and facilitates selective heterodimerization with the MyoD family of gene regulatory factors. The. EMBO J. 14, 1766-72 (1995).

8. Tapscott, S. J. The circuitry of a master switch: Myod and the regulation of skeletal muscle gene transcription. Development 132, 2685-95 (2005).

9. Peng, X. L. et al. MyoD- and FoxO3-mediated hotspot interaction orchestrates super-enhancer activity during myogenic differentiation. Nucleic Acids Res. 45, 8785-805 (2017).

10. Weintraub, H. et al. Activation of muscle-specific genes in pigment, nerve, fat, liver, and fibroblast cell lines by forced expression of MyoD. Proc. Natl Acad. Sci. USA 86, 5434-8 (1989).

11. Buckingham, M. \& Rigby, P. W. Gene regulatory networks and transcriptional mechanisms that control myogenesis. Dev. cell 28, 225-38 (2014).

12. Zammit, P. S. Function of the myogenic regulatory factors Myf5, MyoD, Myogenin and MRF4 in skeletal muscle, satellite cells and regenerative myogenesis. Semin. Cell Dev. Biol. 72, 19-32 (2017).

13. Kohli, R. M. \& Zhang, Y. TET enzymes, TDG and the dynamics of DNA demethylation. Nature 502, 472-9 (2013).

14. Ito, S. et al. Role of Tet proteins in $5 \mathrm{mC}$ to $5 \mathrm{hmC}$ conversion, ES-cell selfrenewal and inner cell mass specification. Nature 466, 1129-33 (2010).

15. Tahiliani, M. et al. Conversion of 5-methylcytosine to 5-hydroxymethylcytosine in mammalian DNA by MLL partner TET1. Science 324, 930-5 (2009).

16. He, Y. F. et al. Tet-mediated formation of 5-carboxylcytosine and its excision by TDG in mammalian DNA. Science 333, 1303-7 (2011).

17. Wu, X. \& Zhang, Y. TET-mediated active DNA demethylation: mechanism, function and beyond. Nat. Rev. Genet. 18, 517-34 (2017).

18. Wu, H. \& Zhang, Y. Reversing DNA methylation: mechanisms, genomics, and biological functions. Cell 156, 45-68 (2014).

19. Melamed P., Yosefzon Y., David C., Tsukerman A. \& Pnueli L. Tet enzymes, variants, and differential effects on function. Front. Cell Dev. Biol. 6, 22 (2018).

20. Kim, M. R., Wu, M. J., Zhang, Y., Yang, J. Y. \& Chang, C. J. TET2 directs mammary luminal cell differentiation and endocrine response. Nat. Commun. 11, 4642 (2020). 
21. Lucarelli, M., Fuso, A., Strom, R. \& Scarpa, S. The dynamics of myogenin sitespecific demethylation is strongly correlated with its expression and with muscle differentiation. J. Biol. Chem. 276, 7500-6 (2001).

22. Palacios, D., Summerbell, D., Rigby, P. W. \& Boyes, J. Interplay between DNA methylation and transcription factor availability: implications for developmental activation of the mouse Myogenin gene. Mol. Cell. Biol. 30, 3805-15 (2010).

23. Zhong, $X$. et al. Ten-eleven translocation-2 (Tet2) is involved in myogenic differentiation of skeletal myoblast cells in vitro. Sci. Rep. 7, 43539 (2017).

24. Horsley, V., Jansen, K. M., Mills, S. T. \& Pavlath, G. K. IL-4 acts as a myoblast recruitment factor during mammalian muscle growth. Cell 113, 483-94 (2003).

25. Teng, S. et al. Phospholipase D1 facilitates second-phase myoblast fusion and skeletal muscle regeneration. Mol. Biol. Cell 26, 506-17 (2015).

26. Manandhar, D. et al. Incomplete MyoD-induced transdifferentiation is associated with chromatin remodeling deficiencies. Nucleic Acids Res 45, 11684-99 (2017).

27. Gu, T. P. et al. The role of Tet3 DNA dioxygenase in epigenetic reprogramming by oocytes. Nature 477, 606-610 (2011).

28. Zhang, T. et al. Phosphorylation of TET2 by AMPK is indispensable in myogenic differentiation. Epigenetics Chromatin 12, 32 (2019).

29. Oikawa, Y. et al. The methyl-CpG-binding protein CIBZ suppresses myogenic differentiation by directly inhibiting myogenin expression. Cell Res. 21, 1578-90 (2011).

30. Xu, Y. et al. Tet3 CXXC domain and dioxygenase activity cooperatively regulate key genes for Xenopus eye and neural development. Cell 151, 1200-13 (2012).

31. Ko, M. et al. Modulation of TET2 expression and 5-methylcytosine oxidation by the CXXC domain protein IDAX. Nature 497, 122-6 (2013).

32. Sartorelli, V. \& Puri, P. L. Shaping gene expression by landscaping chromatin architecture: lessons from a master. Mol. Cell 71, 375-88 (2018).
33. Cao, Y. et al. Genome-wide MyoD binding in skeletal muscle cells: a potentia for broad cellular reprogramming. Dev. Cell 18, 662-74 (2010).

34. Feng $X$. et al. Dual function of VGLL4 in muscle regeneration. EMBO J. 38, e101051 (2019)

35. $\mathrm{Fu}, \mathrm{X}$. et al. Combination of inflammation-related cytokines promotes longterm muscle stem cell expansion. Cell Res. 25, 1082-3 (2015).

36. Gallot Y. S., Hindi S. M., Mann A. K. \& Kumar A. Isolation, culture, and staining of single myofibers. Bio Protoc. 6, e1942 (2016).

37. Hu, P., Geles, K. G., Paik, J. H., DePinho, R. A. \& Tjian, R. Codependent activators direct myoblast-specific MyoD transcription. Dev. Cell 15, 534-46 (2008).

38. Hu, P., Wu, S. \& Hernandez, N. A minimal RNA polymerase III transcription system from human cells reveals positive and negative regulatory roles for CK2. Mol. Cell 12, 699-709 (2003).

39. Rohde, C., Zhang, Y., Reinhardt, R. \& Jeltsch, A. BISMA—fast and accurate bisulfite sequencing data analysis of individual clones from unique and repetitive sequences. BMC Bioinforma. 11, 230 (2010).

40. Wingett, S. W. \& Andrews, S. FastQ Screen: A tool for multi-genome mapping and quality control. F1000Res. 7, 1338 (2018).

41. Lu, L. et al. Genome-wide survey by ChIP-seq reveals YY1 regulation of lincRNAs in skeletal myogenesis. EMBO J. 32, 2575-88 (2013).

42. Merritt, E. K. et al. Heightened muscle inflammation susceptibility may impair regenerative capacity in aging humans. J. Appl Physiol. 115, 937-48 (2013)

43. Trapnell, C. et al. Differential analysis of gene regulation at transcript resolution with RNA-seq. Nat. Biotechnol. 31, 46-53 (2013).

44. Jiang P. et al. Methy-Pipe: an integrated bioinformatics pipeline for whole genome bisulfite sequencing data analysis. PLOS ONE. 9, e100360 (2014).

45. Langmead, B. \& Salzberg, S. L. Fast gapped-read alignment with Bowtie 2. Nat. Methods 9, 357-9 (2012).

46. Zhang, Y. et al. Model-based analysis of ChIP-Seq (MACS). Genome Biol. 9, R137 (2008). 